University of Wollongong

Research Online

Faculty of Informatics - Papers (Archive)

Faculty of Engineering and Information

Sciences

May 2002

\title{
Frame bounds for biorthogonal cosine-modulated filter banks
}

Alfred Mertins

University of Wollongong, mertins@uow.edu.au

Follow this and additional works at: https://ro.uow.edu.au/infopapers

Part of the Physical Sciences and Mathematics Commons

\section{Recommended Citation}

Mertins, Alfred: Frame bounds for biorthogonal cosine-modulated filter banks 2002.

https://ro.uow.edu.au/infopapers/96

Research Online is the open access institutional repository for the University of Wollongong. For further information contact the UOW Library: research-pubs@uow.edu.au 


\title{
Frame bounds for biorthogonal cosine-modulated filter banks
}

\author{
Abstract \\ We derive explicit expressions for the eigenvalues of the frame operator for cosine-modulated filter \\ banks. The filter banks may be critically sampled or oversampled by an integer factor. The analysis of low- \\ delay, biorthogonal filter banks shows that prototypes solely designed to minimize the stopband energy \\ may lead to wide open frames and thus to an undesirable numerical behavior. Because the computational \\ cost of determining the frame bounds with the proposed method is very low, we can directly use the \\ bounds during prototype optimization and obtain prototypes with minimum stopband energy under the \\ condition of fixed frame bounds. \\ Disciplines \\ Physical Sciences and Mathematics

\section{Publication Details} \\ This article was published as: Mertins, A, Frame bounds for biorthogonal cosine-modulated filter banks, \\ Proceedings IEEE International Conference on Acoustics, Speech and Signal Processing, May 13 2002, 2, \\ 1241-1244. Copyright IEEE 2002.
}




\title{
FRAME BOUNDS FOR BIORTHOGONAL COSINE-MODULATED FILTER BANKS
}

\author{
Alfred Mertins \\ University of Wollongong, \\ School of Electrical, Computer, and Telecommunications Engineering, \\ Wollongong, NSW 2522, Australia. Email: mertins@uow.edu.au.
}

\begin{abstract}
In this paper, we derive explicit expressions for the eigenvalues of the frame operator for cosine-modulated filter banks. The filter banks may be critically sampled or oversampled by an integer factor. The analysis of low-delay, biorthogonal filter banks shows that prototypes solely designed to minimize the stopband energy may lead to wide open frames and thus to an undesirable numerical behavior. Because the computational cost of determining the frame bounds with the proposed method is very low, we can directly use the bounds during prototype optimization and obtain prototypes with minimum stopband energy under the condition of fixed frame bounds.
\end{abstract}

\section{INTRODUCTION}

Biorthogonal, low-delay, cosine-modulated filter banks have been studied in [1-7]. Such designs are attractive, because they allow to choose the overall system delay independent of the lengths of the filters involved. A collection of general PR conditions for critical sampling can be found in [6]. Design methods for FIR oversampled filter banks were considered in $[5,8]$.

The quality criterion commonly used in the design of both paraunitary and biorthogonal filter banks is the minimization of the stopband energy of the prototype [4-7]. However, for biorthogonal filter banks good properties of a prototype (flat passband, high stopband attenuation) do not necessarily translate into good properties of the modulated filters. In this paper, we will study this phenomenon using frame analysis, which is a powerful tool to assess the properties of filter banks and transforms [8-12]. Based on the general methods proposed in [10] for determining frame bounds through an eigen-analysis of a frequency-dependent matrix, known as the frame operator, we derive new expressions for the frame bounds which are very easy and efficient to compute. In particular, we will derive explicit expressions for the eigenvalues of the frame operator. For the special case of critically sampled filter banks we will present an even more efficient method that finds the bounds directly from Fourier transforms of the autocorrelation sequences of the prototype's polyphase components. Because the computational cost of determining the frame bounds with the proposed method is very low, we can directly use the bounds during prototype optimization and obtain prototypes with minimum stopband energy under the condition of fixed frame bounds.

Notation: Matrices and vectors are printed in boldface. $I_{M}$ and $J_{M}$ denote the $M \times M$ identity and counter identity matrices, respectively. The term diag $[\cdot]$ denotes the formation of

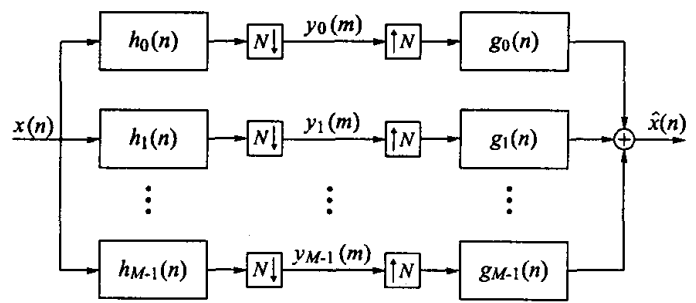

Fig. 1. $M$-band analysis and synthesis filter banks with sampling rate ratio $N$

a diagonal matrix. $\|\cdot\|$ means the Euclidean norm of a vector. $\mathbb{Z}$ is the set of all integers, and $\mathbb{R}$ is the set of all real numbers. The asterisk $*$ denotes convolution.

\section{COSINE-MODULATED FILTER BANKS}

We consider cosine-modulated filter banks where the analysis and synthesis filters are derived from the same prototype $p(n)$. Fig. 1 shows the filter bank in direct form where $h_{k}(n)$ and $g_{k}(n), k=0, \ldots, M-1$ denote the impulse responses of the analysis and synthesis filters, respectively. $M$ is the number of channels, and $N$ is the ratio between the sampling rate at the input and in the subbands. $M$ may be even or odd, but the oversampling factor $L=M / N$ is supposed to be an integer. The filters $h_{k}(n)$ and $g_{k}(n)$ are derived as [5]

$$
h_{k}(n)=\frac{1}{\sqrt{L}} p(n) c_{1, k}(n), g_{k}(n)=\frac{1}{\sqrt{L}} p(n) c_{2, k}(n)
$$

with

$$
\begin{aligned}
& c_{1, k}(n)=\sqrt{\frac{2}{M}} \cos \left[\frac{\pi}{M}\left(k+\frac{1}{2}\right)\left(n-\frac{D}{2}\right)+(-1)^{k} \pi / 4\right], \\
& c_{2, k}(n)=\sqrt{\frac{2}{M}} \cos \left[\frac{\pi}{M}\left(k+\frac{1}{2}\right)\left(n-\frac{D}{2}\right)-(-1)^{k} \pi / 4\right] .
\end{aligned}
$$

$D$ is the overall delay of the analysis/synthesis system and is assumed to be of the form $D=2 s M+2 M-1$ where $s$ is an integer.

The analysis and synthesis polyphase matrices for the above filter bank, denoted as $E^{(L)}(z)$ and $R^{(L)}(z)$, respectively, can be expressed as [5]

$$
\boldsymbol{E}^{(L)}(z)=\frac{1}{\sqrt{L}} C_{1}\left[\begin{array}{c}
\boldsymbol{P}_{0}\left(z^{2 L}\right) \\
z^{-1} \boldsymbol{P}_{1}\left(z^{2 L}\right) \\
\vdots \\
z^{-(2 L-1)} \boldsymbol{P}_{2 L-1}\left(z^{2 L}\right)
\end{array}\right]
$$




$$
\begin{array}{r}
\boldsymbol{R}^{(L)}(z)=\frac{1}{\sqrt{L}\left[z^{-(2 L-1)} J_{N} P_{2 L-1}\left(z^{2 L}\right) J_{N}\right.} \\
z^{-(2 L-2)} J_{N} P_{2 L-2}\left(z^{2 L}\right) J_{N}, \ldots \\
\left.\ldots, J_{N} P_{0}\left(z^{2 L}\right) J_{N}\right] C_{2}^{T}
\end{array}
$$

where the superscript ${ }^{(L)}$ indicates the oversampling factor. $C_{1}$ and $C_{2}$ in (3) and (4) are $M \times 2 M$ cosine modulation matrices given by

$$
\left[C_{1}\right]_{k, n}=c_{1, k}(n), \quad\left[C_{2}\right]_{k, 2 M-1-n}=c_{2, k}(n)
$$

with $k=0,1, \ldots, M-1$ and $n=0,1, \ldots, 2 M-1$. The matrices $P_{k}\left(z^{2 L}\right)$ are defined as

$$
\begin{aligned}
P_{k}\left(z^{2 L}\right)= & \operatorname{diag}\left[P_{k N}\left(-z^{2 L}\right), P_{k N+1}\left(-z^{2 L}\right)\right. \\
& \left.\ldots, P_{k N+N-1}\left(-z^{2 L}\right)\right], \quad k=0,1, \ldots, 2 L-1
\end{aligned}
$$

where $P_{j}(z)=\sum_{\ell} p(2 \ell M+j) z^{-\ell}$. With $R^{(L)}(z)$ and $E^{(L)}(z)$ the perfect reconstruction condition on the filter bank can be formulated as $R^{(L)}(z) E^{(L)}(z)=z^{-2 s L-2 L-1} I_{N}$, which finally leads to the following PR conditions on the prototype for $L$ times oversampled, biorthogonal, cosine-modulated filter banks [5]:

$$
\begin{array}{r}
\sum_{\ell=0}^{2 L-1} P_{k+\ell N}(z) P_{2 M-1-k-\ell N}(z)=L z^{-s} \\
k=0, \ldots, N-1 .
\end{array}
$$

\section{DEFINITION OF FRAMES}

Let $h_{k, m}, k=0,1, \ldots, M-1, m \in \mathbb{Z}$ be vectors containing the filter coefficients $h_{k}(m M-n)$ and let $x$ be a vector containing the input samples $x(n)$. Assuming that $x \in \ell^{2}(\mathbb{Z})$ the following inequality can be stated:

$$
A\|x\|^{2} \leq \sum_{k=0}^{M-1} \sum_{m=-\infty}^{\infty}\left|\left\langle\boldsymbol{x}, h_{k, m}\right\rangle\right|^{2} \leq B\|x\|^{2} \forall x \in \ell^{2}(\mathbb{Z})
$$

with some $A, B \in \mathbb{R}$. If $A>0$ and $B<\infty$ the values $A$ and $B$ are called frame bounds and the set $h_{k, m}$ is called a frame for $\ell^{2}(\mathbb{Z})$. The frame bounds themselves can be seen as indicators of the numerical properties of the filter bank. They have proven to be a useful tool for characterizing the properties of filter banks [8-12] and have especially been used to analyze oversampled filter banks, including cosine-modulated ones [8].

If the set $\boldsymbol{h}_{k, m}$ constitutes a frame then there exists a corresponding PR synthesis filter bank with filters $g_{k, m}$ that also constitutes a frame with frame bounds $A^{\prime}$ and $B^{\prime}$ such that

$$
A^{\prime}\|x\|^{2} \leq \sum_{k=0}^{M-1} \sum_{m=-\infty}^{\infty}\left|\left\langle x, g_{k, m}\right\rangle\right|^{2} \leq B^{\prime}\|x\|^{2} \forall x \in \ell^{2}(\mathbb{Z})
$$

In the special case that $A=B=1$ the frame is called a tight frame. Then the filter bank is paraunitary, the vectors $h_{k, m}$ form an orthonormal basis for $\ell^{2}(\mathbb{Z})$, and the inequality (8) simply reduces to Parseval's identity $\|x\|^{2}=$ $\sum_{k=0}^{M-1} \sum_{m=-\infty}^{\infty}\left|\left\langle\boldsymbol{x}, \boldsymbol{h}_{k, m}\right\rangle\right|^{2}$. In general, the smaller the ratios $B / A$ and $B^{\prime} / A^{\prime}$ the better the numerical properties of the filter bank will be. If $B / A$ and $B^{\prime} / A^{\prime}$ are close to one then the filter bank can be regarded as being almost paraunitary and Parseval's identity may be used without much error when relating the energy of the subband signals to the energy of the input or output signal of a filter bank [12]. Having almost paraunitary filter banks is particularly useful in source coding where operational rate-distortion algorithms are to be applied in the subband domain and exact paraunitaryness cannot be achieved because of other requirements such as linear phase or low delay.

\section{COMPUTATION OF FRAME BOUNDS}

General algorithms for the computation of frame bounds have been described in $[10,12]$. We will follow the method in [10] and use it to derive explicit formulas for the eigenvalues of the frame operator for cosine-modulated filter banks. Due to space limitations we only consider the computation of frame bounds for even $N$. However, odd $N$ can be treated in a similar way.

Let $\lambda_{k}(\omega), k=0,1, \ldots, N-1$ be the eigenvalues of

$$
\boldsymbol{S}(z)=\left[\boldsymbol{E}^{(L)}\left(z^{-1}\right)\right]^{T} \boldsymbol{E}^{(L)}(z) .
$$

The frame bounds $A$ and $B$ are then given by [8]

$$
\begin{gathered}
A=\underset{\omega \in[0,2 \pi), k=0,1, \ldots, N-1}{\operatorname{essinf}} \lambda_{k}(\omega), \\
B=\underset{\omega \in[0,2 \pi), k=0,1, \ldots, N-1}{\operatorname{ess} \sup _{k}} \lambda_{k}(\omega) .
\end{gathered}
$$

Inserting (3) and (4) into (10) and rewriting the expression obtained using the property

$$
C_{1}^{\mathrm{T}} C_{1}=\left[\begin{array}{cc}
I_{M}+(-1)^{s} J_{M} & 0 \\
0 & I_{M}-(-1)^{s} J_{M}
\end{array}\right]
$$

yields

$$
\begin{aligned}
& S(z)=\frac{1}{L} \sum_{\ell=0}^{2 L-1} P_{\ell}\left(z^{-2 L}\right) P_{\ell}\left(z^{2 L}\right)+ \\
& +(-1)^{s} \frac{1}{L} \sum_{\ell=0}^{L-1} z^{L-1-2 \ell} P_{L-1-\ell}\left(z^{-2 L}\right) J_{N} P_{\ell}\left(z^{2 L}\right) \\
& -(-1)^{s} \frac{1}{L} \sum_{\ell=0}^{L-1} z^{L-1-2 \ell} P_{2 L-1-\ell}\left(z^{-2 L}\right) J_{N} P_{L+\ell}\left(z^{2 L}\right) .
\end{aligned}
$$

Because the matrices $P_{\ell}\left(z^{2 L}\right)$ are diagonal, it turns out that $S(z)$ has non-zero elements on its diagonal and anti diagonal only. Using this fact, we can reduce the task of computing the eigenvalues of $S\left(e^{j \omega}\right)$ to the task of determining the eigenvalues of the $2 \times 2$ matrices

$$
S_{k}\left(e^{j \omega}\right)=\left[\begin{array}{cc}
S_{k, k}\left(e^{j \omega}\right) & S_{k, N-1-k}^{\prime}\left(e^{j \omega}\right) \\
S_{N-1-k, k}^{\prime}\left(e^{j \omega}\right) S_{N-1-k, N-1-k}\left(e^{j \omega}\right)
\end{array}\right]
$$

for $k=0,1, \ldots, \frac{N}{2}-1$ with

$$
S_{k, k}(z)=\frac{1}{L} \sum_{\ell=0}^{2 L-1} P_{k+\ell N}\left(-z^{-2 L}\right) P_{k+\ell N}\left(-z^{2 L}\right)
$$

and

$$
\begin{gathered}
S_{k, N-1-k}^{\prime}(z)=\frac{(-1)^{s}}{L} \sum_{\ell=0}^{L-1} z^{L-1-2 \ell} P_{(L-1-\ell) N+k}\left(-z^{-2 L}\right) \\
-\frac{(-1)^{s}}{L} \sum_{\ell=0}^{L-1} z^{L-1-2 \ell} P_{(2 L-1-\ell) N+k}\left(-z^{-2 L}\right) \\
\times P_{(L+\ell) N+N-1-k}\left(-z^{2 L}\right) .
\end{gathered}
$$


The eigenvalues of $\boldsymbol{S}_{k}\left(e^{j \omega}\right)$ can then be explicitly expressed as

$$
\begin{aligned}
\lambda_{k ; \frac{N}{2}+k}(\omega)= & \frac{1}{2}\left(S_{k, k}\left(e^{j \omega}\right)+S_{N-1-k, N-1-k}\left(e^{j \omega}\right)\right) \\
\pm \frac{1}{2}\left[\left(S_{k, k}\left(e^{j \omega}\right)-\right.\right. & \left.S_{N-1-k, N-1-k}\left(e^{j \omega}\right)\right)^{2}+ \\
& \left.+4\left|S_{k, N-1-k}^{\prime}\left(e^{j \omega}\right)\right|^{2}\right]^{1 / 2}
\end{aligned}
$$

The required terms $S_{k, k}\left(e^{j \omega}\right)$ and $S_{k, N-1-k}^{\prime}\left(e^{j \omega}\right)$ can be computed in a fast way as the discrete-time Fourier transforms of sequences $s_{k, k}(n)$ and $s_{k, N-1-k}^{\prime}(n)$ that are obtained as

$$
s_{k, k}(n)=\frac{1}{L} \sum_{\ell=0}^{2 L-1} \phi_{k+\ell N}(-n) * \phi_{k+\ell N}(n)
$$

and

$$
\begin{aligned}
s_{k, N-1-k}^{\prime}(n)= & \frac{(-1)^{s}}{L} \sum_{\ell=0}^{L-1} \delta(n+L-1-2 \ell) \\
& * \phi_{(L-1-\ell) N+k}(-n) * \phi_{\ell N+N-1-k}(n) \\
& -\frac{(-1)^{s}}{L} \sum_{\ell=0}^{L-1} \delta(n+L-1-2 \ell) \\
& * \phi_{(2 L-1-\ell) N+k}(-n) * \phi_{(L+\ell) N+N-1-k}(n)
\end{aligned}
$$

with

$$
\phi_{k}(n)= \begin{cases}(-1)^{n} p_{k}\left(\frac{n}{2 L}\right), & \text { if } \frac{n}{2 L} \in \mathbb{Z}, \\ 0, & \text { otherwise. }\end{cases}
$$

In practice, an FFT algorithm and zero padding can be used to compute $S_{k, k}\left(e^{j \omega}\right)$ and $S_{k, N-1-k}^{\prime}\left(e^{j \omega}\right)$ from $s_{k, k}(n)$ and $s_{k, N-1-k}^{\prime}(n)$ on a fine frequency grid.

To obtain the frame bounds for the synthesis filters we may carry out a similar derivation as for the analysis side, based on the eigenvalues of $\boldsymbol{T}\left(e^{j \omega}\right)=\boldsymbol{R}^{(L)}\left(e^{j \omega}\right)\left[\boldsymbol{R}^{(L)}\left(e^{j \omega}\right)\right]^{H}$. For even $N$, as considered here, this yields $A^{\prime}=A$ and $B^{\prime}=B$.

Frame Bounds for Critical Sampling. Analyzing the product $\lambda_{k}^{\prime}(\omega) \lambda_{N / 2+k}^{\prime}(\omega)$ with $L=1$ and thus with $N=M$ under consideration of (15), (16) and (7) yields

$$
\lambda_{k}(\omega) \lambda_{M / 2+k}(\omega)=1 \quad \forall \omega
$$

This interesting relationship immediately implies that

$$
A B=1
$$

for critically sampled PR cosine-modulated filter banks. Equation (21) is also the key to further simplification of the computation of the frame bounds. From (21), (17), and (15) (for $z=$ $e^{j \omega}$ and under consideration of $P_{k}\left(-e^{-j 2 \omega)}\right) P_{k}\left(-e^{j 2 \omega)}\right)=$ $\left.\left|P_{k}\left(e^{j(2 \omega+\pi)}\right)\right|^{2}\right)$ it follows that

$$
\lambda_{M / 2+k}(\omega)=A_{k}(\omega)+\sqrt{A_{k}^{2}(\omega)-1}
$$

with

$$
\begin{aligned}
& A_{k}(\omega)=\frac{1}{2}\left[\left|P_{k}\left(e^{j(2 \omega+\pi)}\right)\right|^{2}+\left|P_{M+k}\left(e^{j(2 \omega+\pi)}\right)\right|^{2}\right. \\
& \left.\quad+\left|P_{M-1-k}\left(e^{j(2 \omega+\pi)}\right)\right|^{2}+\left|P_{2 M-1-k}\left(e^{j(2 \omega+\pi)}\right)\right|^{2}\right] .
\end{aligned}
$$

For further simplification we define

$$
A_{k}^{\prime}(\omega)=A_{k}\left(\frac{\omega-\pi}{2}\right)
$$

which is the discrete-time Fourier transform of the sequence

$$
\begin{aligned}
a_{k}^{\prime}(n)= & \frac{1}{2}\left[p_{k}(n) * p_{k}(-n)+p_{M+k}(n) * p_{M+k}(-n)\right. \\
& +p_{M-1-k}(n) * p_{M-1-k}(-n) \\
& \left.+p_{2 M-1-k}(n) * p_{2 M-1-k}(-n)\right] .
\end{aligned}
$$

The frame bounds finally amount to

$$
B=\beta+\sqrt{\beta^{2}-1}, \quad A=1 / B=\beta-\sqrt{\beta^{2}-1}
$$

with

$$
\beta=\underset{\omega \in[0, \pi)}{\operatorname{ess} \sup } \underset{k=0,1, \ldots, M / 2-1}{ } A_{k}^{\prime}(\omega)
$$

\section{EXAMPLES}

We consider $M=8$ channels, a delay of $D=15$ taps, filters of length 48 , and critical sampling. Prototypes were designed to minimize the stopband energy

$$
\Phi=\int_{\omega_{s}}^{\pi}\left|P\left(e^{j \omega}\right)\right|^{2} d \omega
$$

under the constraint (7), using the lifting structure of [13] for parameterization. The stopband edge frequency for the prototypes was chosen as $\omega_{s}=\pi / M$. To design biorthogonal filter banks with controlled frame bounds the constraint $B \leq B_{\max }$ was added during optimization. Due to (22) this implies that $A \geq 1 / B_{\max }$ for the case of critical sampling. For the constrained optimization the Matlab routine fmincon has been used.

First we consider a prototype that was designed to minimize (29) under the PR constraint for critical sampling, without imposing frame bounds. The frequency response of the prototype is shown in Fig. 2(a), and the frequency responses of the resulting analysis filters are depicted in Fig. 2(b). One can see that the near-ideal frequency response of the prototype does not translate into near-ideal modulated filters. The first and last filters, $h_{0}(n)$ and $h_{M-1}(n)$, show an undesirable passband behavior that is significantly different from that of the prototype. For this filter bank the stopband energy is $\Phi=0.06$ and the frame bounds amount to $A=0.31, B=3.27$. This means that the energy of the input and output signals of the filter bank may be different from the subband energy by a factor between 0.31 and 3.27, depending on the actual signal. Thus, although the filter bank constitutes a frame and provides PR it is not well suited for schemes that rely on the assumption that the subband energy is close to the input/output signal energy. Also, quantization noise introduced in the subbands may arrive at the output with an amplification of up to 3.27 (in terms of the noise power).

We now consider the design of a prototype that minimizes (29) under the condition $B \leq 1.1$ for $M=8, D=15$, and critical sampling. Fig. 3 shows the frequency responses of the prototype and the analysis filters for $B=1 / A=1.1$. As the graphs in Fig. 3(b) show, all analysis filters are almost ideally frequency translated versions of the prototype. The value $B=$ 1.1 indicates a relatively tight frame, so that the assumption of a near-unitary behavior can be justified in applications such as signal compression. 


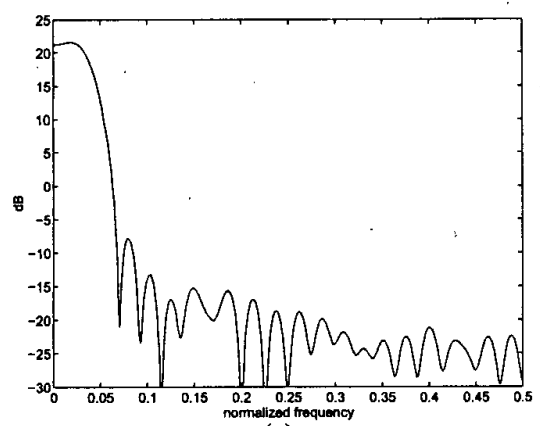

(a)

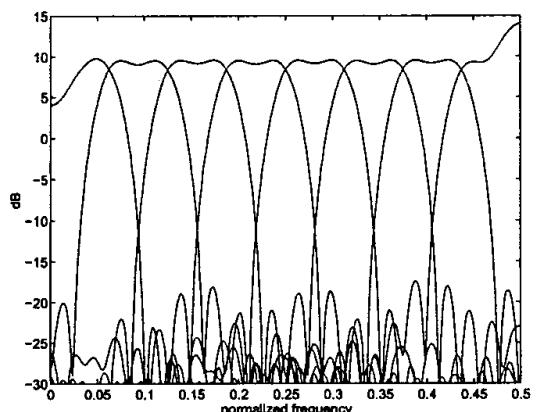

(b)

Fig. 2. Frequency responses of 8-channel filter bank, designed to minimize the stopband energy. (a) prototype; (b) analysis filters.

\section{CONCLUSIONS}

Explicit expressions for the eigenvalues of the frame operator for cosine-modulated filter banks have been presented, which allow an efficient computation of frame bounds directly from the prototype's polyphase components. The analysis of lowdelay, biorthogonal filter banks showed that prototypes solely designed to minimize the stopband energy may lead to wide open frames and thus to an undesirable numerical behavior. By incorporating a frame analysis directly into the prototype design process, however, the numerical properties of the resulting filter banks can be directly controlled.

\section{REFERENCES}

[1] K. Nayebi, T.P. Barnwell III, and M. J. T. Smith, "Low delay FIR filter banks: Design and evaluation," IEEE Trans. Signal Processing, vol. SP-42, pp. 24-31, Jan. 1994.

[2] G. Strang and T. Nguyen, Wavelets and Filter Banks, Wellesley-Cambridge Press, Wellesley, MA, 1996.

[3] G. D. T. Schuller and M. J. T. Smith, "A new framework for modulated perfect reconstruction filter banks," IEEE Trans. Signal Processing, vol. 44, no. 8, pp. 1941-1954, Aug. 1996.

[4] T. Q. Nguyen and P. N. Heller, "Biorthogonal cosinemodulated filter banks," in Proc. IEEE Int. Conf. Acoust., Speech, Signal Processing, Atlanta, USA, May 1996.

[5] J. Kliewer and A. Mertins, "Oversampled cosine-modulated filter banks with arbitrary system delay," IEEE Trans. Signal Processing, vol. 46, no. 4, pp. 941 - 955, April 1998.

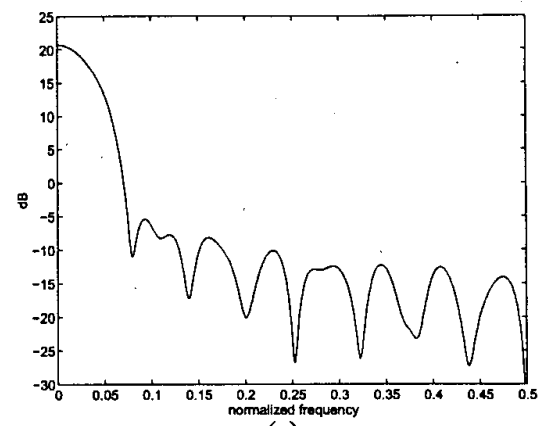

(a)

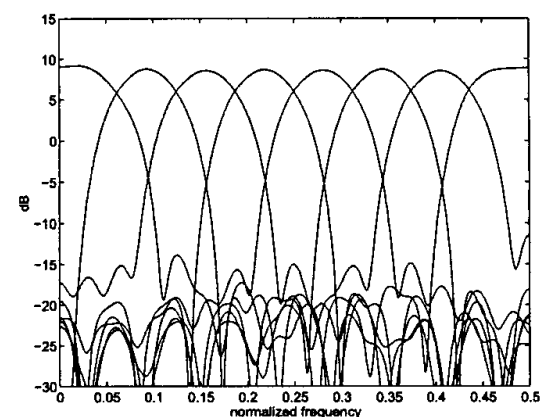

(b)

Fig. 3. Frequency responses of 8-channel filter bank, designed to minimize the stopband energy under the condition of $B=1.1$. (a) prototype; (b) analysis filters.

[6] P. N. Heller, T. Karp, and T. Q. Nguyen, "A general formulation for modulated filter banks," IEEE Trans. Signal Processing, vol. 47, pp. 986-1002, Apr. 1999.

[7] F. Argenti and E. Del Re, "Design of biorthogonal m-channel cosine-modulated fir/iir filter banks," IEEE Trans. Signal Processing, vol. 48, no. 3, pp. 876-881, Mar. 2000.

[8] H. Bölcskei and F. Hlawatsch, "Oversampled cosine modulated filter banks with perfect reconstruction," IEEE Trans. Circuits and Systems II, vol. 45, no. 8, pp. 1057-1071, Aug. 1998.

[9] I. Daubechies, Ten Lectures on Wavelets, SIAM, 1992.

[10] H. Bölcskei, F. Hlawatsch, and H. G. Feichtinger, "Frametheoretic analysis of oversampled filter banks," IEEE Trans. Signal Processing, vol. 46, no. 12, pp. 3256-3268, Dec. 1998.

[11] G. Cvetković and M. Vetterli, "Oversampled filter banks," IEEE Trans. Signal Processing, vol. 46, no. 5, pp. 12451255 , May 1998.

[12] F. Moreau de Saint-Martin, P. Siohan, and A. Cohen, "Biorthogonal filterbanks and energy preservation property in image compression," IEEE Trans. Image Processing, vol. 8, no. 2, pp. 168-178, Feb. 1999.

[13] T. Karp, A. Mertins, and G. Schuller, "Efficient biorthogonal cosine-modulated filter banks," EURASIP Signal Processing, vol. 81, no. 5, pp. 997-1016, May 2001. 